\title{
Characterization of a parietal lesion detected by diffusion weighted MRI, NaF18-PET/ CT and CT
}

\author{
Aung Zaw Win, Carina Mari Aparici \\ Division of Nuclear Medicine, Department of Radiology, San Francisco VA Medical Center, San Francisco, California, USA \\ Correspondence: Aung Zaw Win. Address: 4150 Clement Street, San Francisco, CA 94121. \\ Email: aungzwin@gmail.com.
}

Received: April 4, 2013

DOI : $10.5430 /$ jbgc.v3n4p43
Online Published: July 5, 2013

URL: http://dx.doi.org/10.5430/jbgc.v3n4p43

\begin{abstract}
It is very important to accurately characterize skull lesions so that appropriate treatment decisions can be made. False positive readings may lead to unnecessary surgical intervention or radiation. We present a case of a parietal lesion seen on Diffusion weighted (DW) MRI, and further characterized with NaF18-PET/CT and CT in a 69 year old male. This is the first case to report the use of DW MRI, NaF18-PET/CT bone scan and CT in the diagnosis of a skull lesion. DW MRI is known to have high specificity to detect malignant lesions in the skull. If a similar case such as ours in encountered in everyday clinical practice, a differential diagnosis of a benign etiology should be considered.
\end{abstract}

\section{Key words}

Diffusion weighted MRI, Parietal lesion, NaF18-PET/CT

\section{I ntroduction}

To our knowledge, this is the first case to report the use of both DW MRI and NaF18-PET/CT bone scan in the diagnosis of a skull lesion. Prostate, breast and lung primaries account for the majority of skull metastases. Metastatic tumors of skull are more common than primary skull tumors. Tumors usually spread to bone hematogenously. Bone metastases may be asymptomatic or may produce pain, swelling, and pathologic fracture ${ }^{[1]}$. DW MRI method relies on the diffusion of water molecules across cells and tissues for imaging ${ }^{[2]}$. It can detect physiological abnormalities even before structural changes are apparent. This technique has great versatility because it can be used for making a primary diagnosis and prognosis prediction, following the treatment response after chemotherapy or radiation and restaging of tumors. Cancerous lesions have higher cell density, fibrous tissue and areas of necrosis compared to surrounding normal tissues ${ }^{[2]}$. Thus, tumors present a greater barrier to water diffusion than normal tissues. Neoplastic lesions can show up as high intensity lesions of DW MRI. The advantages of DW MRI include quick imaging time, noninvasive protocol, no radiation exposure and no need for contrast. However, there are limitations for this type of imaging. It is very prone to artifacts such as motion and breathing artifacts ${ }^{[2]}$. The standard 1.5 T MRI used in our case is best for DW MRI protocol because it can reduce the background noises. Moreover, sclerotic lesions such as metastasis from prostate cancer do not show up as high intensity lesions ${ }^{[2]}$. NaF18-PET/CT can detect bone metastases based on the osteoblastic reaction. It has the advantage of being able to image the whole body in one exam. It has higher sensitivity than Tc-99m MDP bone scan and it can more accurately differentiate between benign and malignant lesions ${ }^{[3]}$. 


\section{Case report}

This case report was approved by the institutional review board. This is a 69 year old white male without any history of cancer but with a history of chronic smoking, alcohol abuse, osteopenia, migraine headaches, left sided weakness, myoclonus, intermittent loss of consciousness and frequent falls. He denied any urinary or bowel incontinence but he had 1 recent episode of dyssarthia. In addition to this, he had tinnitus and gait instability. His family history is positive for cancer. He had an MRI exam to evaluate the left sided weakness and it found a right parietal calvarial lesion measuring $1.1 \mathrm{~cm}$, which was suspicious for metastasis (see Figure 1a, 1b). Two days later, the patient underwent a DW MRI to further evaluate the previous findings (see Figure 1c). The DW MRI showed an enhancing $1.1 \mathrm{~cm}$ right parietal lesion with associated reduced diffusion. This lesion was again regarded as highly suspicious for metastasis. There was no evidence of stroke on DW MRI. Complete blood count (CBC), calcium and vitamin B12 levels were within normal limits. Serum and urine protein electrophoresis was negative for immunoglobulins. Three days later, our patient had a head CT and it showed a $6 \times 10 \mathrm{~mm}$ lytic right parietal lesion which extended to the inner table of the calvarium (see Figure 1d). One day later, the patient had a whole body NaF18-PET/CT bone scan and it showed minimally increased osteoblastic activity within the right parietal calvarium, which corresponded to the abnormal focus seen on the recent MRI (see Figure 1e, Figure 2). Five months later, he had a head CT to assess the stability of the lytic skull lesion (see Figure 1f). The most recent head CT showed no interval change in size for the lytic right parietal lesion with no new lesions identified.
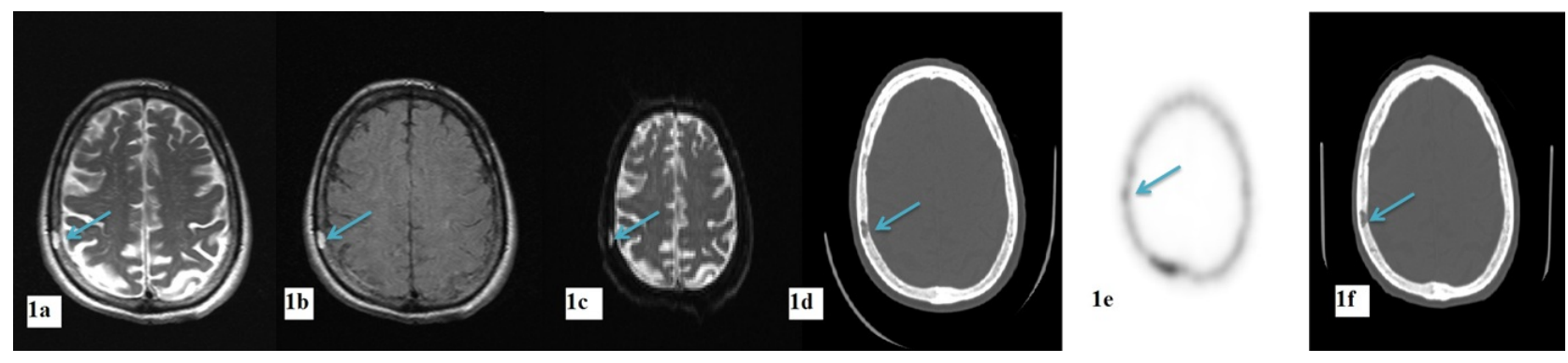

Figure 1. The imaging exams are done in chronological order from left to right. The arrows show the focal lesion on right parietal bone. (a, b) T2 weighted MRI image. (c) DW MR image. (d) first CT image. (e) NaF18 bone scan of calvarium. (f) last CT image.

\section{Discussion}

DW MRI is very specific for detecting strokes and metastases. A study by Nemeth et al reported $100 \%$ specificity for DW MRI in detecting skull metastasis ${ }^{[4]}$. It was found that DW MRI was comparable to or more sensitive than FDG-PET/ $\mathrm{CT}^{[5,6]}$. Moreover, Mosavi et al found that whole body DWI MRI has higher specificity than NaF 18 PET/CT for detection of bone metastases caused by prostate cancer ${ }^{[7]}$. In the skeleton, the uptake of FDG occurs directly into tumor cells, whereas the uptake of fluoride ion depends of skeletal reaction to tumor, with accumulation on the mineralizing surface of bone rather than in the tumor itself ${ }^{[8]}$. Sclerotic metastases are relatively acellular and lower volumes of viable tumor tissue have lower glycolytic rates ${ }^{[8]}$. In prostate cancer with mainly sclerotic skeletal metastases, FDG-PET has low sensitivity ${ }^{[8]}$. In breast cancer, FDG-PET has a high false negative rate ${ }^{[8]}$. This may be because of different affinity of lytic and sclerotic lesions for FDG, both of which may occur in breast cancer. FDG PET produced more false-negative findings for bone metastases than for non-osseous metastases because of the low uptake of FDG by bone ${ }^{[9]}$. NaF18 PET can more correctly identify bone lesions as benign or malignant ${ }^{[8]}$.

Our patient had both DW MRI and bone scan and the lytic right parietal lesion was detected on both exams. Lesions with focal fluoride ion uptake are associated with malignancy while a diffuse uptake indicates a benign condition. Furthermore, benign lesions can have the appearance of hyperintensity on DW MRI because they can contain fat cells, pus, edema, cyst, scar tissue, fibrous tissue and necrotic tissue and this can hinder the movement of water molecules. On MRI, age related 
changes are seen specifically in parietal and occipital bones in the skull ${ }^{[10]}$. Our patient is 69 years old and he is in the age group where changes are pronounced in the marrow fat composition and bone thickness. Yet, there was a focus of lytic lesion in the parietal bone, distinct from the rest of the calvarium. MRI appearance of fibrous dysplasias often resemble that of tumors and they can show up as high intensity lesions on MRI ${ }^{[11]}$. Primary skull neoplasms such as fibrosarcoma and osteosarcoma can also present as high intensity lesions. Osteoblastomas, which have a preference for frontal and parietal bones in the skull, can present as a single lesion and they can be very slow growing ${ }^{[12]}$. Likewise, other benign diseases of the skull such as Paget's disease can have high MRI signal intensity depending on the stage of the disease ${ }^{[13]}$. Infectious abscesses and osteomyelitis can have similar high MRI signal intensity ${ }^{[14]}$. The neurological symptoms remained unexplained in our patient. Even though DW MRI has a high specificity in detecting metastasis to the skull, the right parietal lesion in this case was considered to be a false positive lesion. In the end, based on the findings of the last CT and the history of frequent falls, the right parietal lesion was deemed benign in etiology.

Figure 2. NaF18-PET/CT whole body bone scan, showing no evidence of metastatic disease. There is evidence of rib trauma, facet joint disease in the cervical and lumbar spine and trauma in the right occipital bone. The arrows show the focal lytic lesion on the right parietal bone. (a) whole body MIP image. (b) CT image for attenuation correction and anatomic localization. (c) NaF18 bone scan of calvarium showing mildy increased osteoblastic activity in right parietal bone. (d) hybrid NaF PET/CT image.

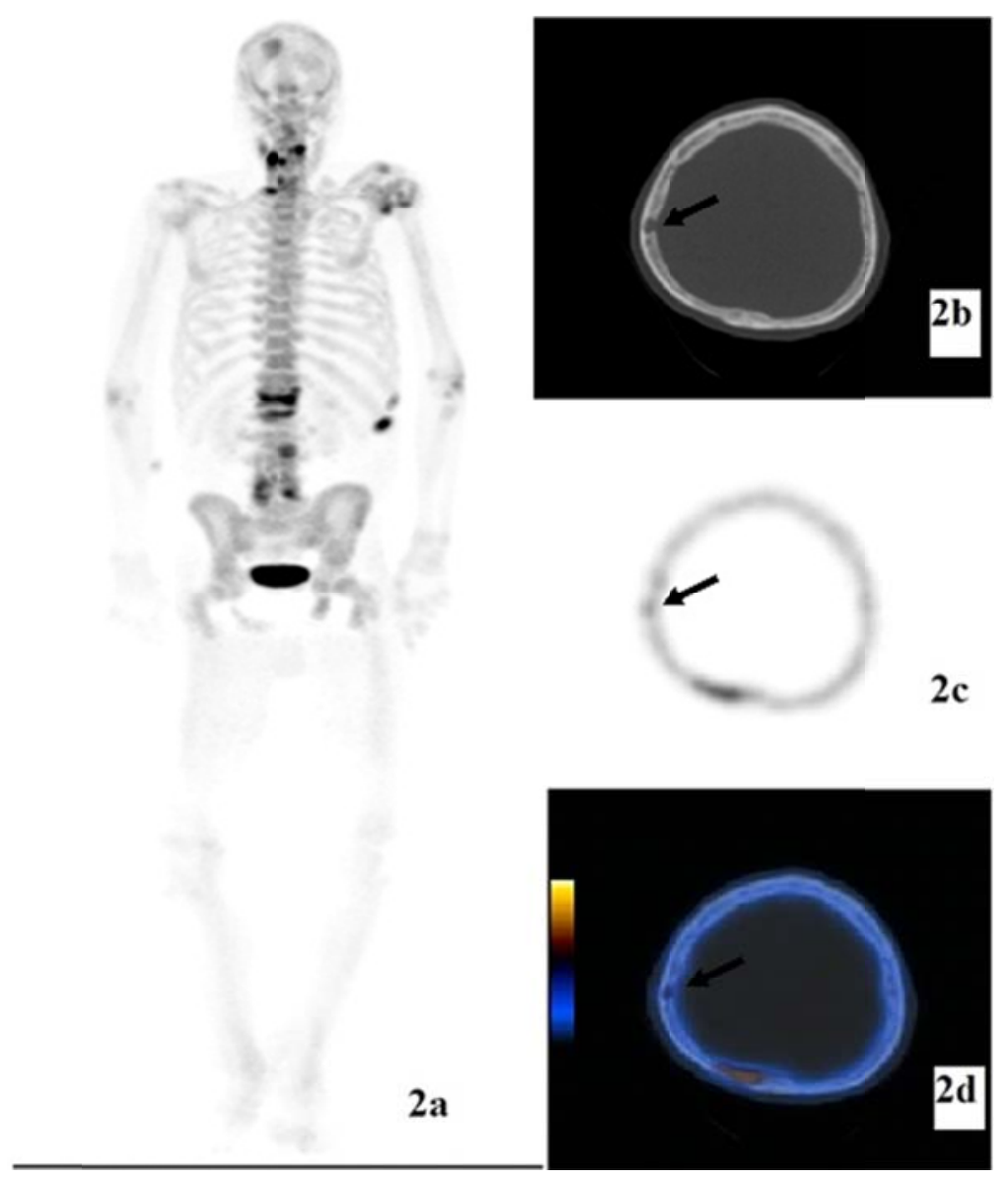

\section{Conclusion}

Nonspecific high intensity lytic skull lesions can become diagnostic dilemmas. The prognosis of a patient depends on choosing the right therapeutic strategy. Due to its high sensitivity, specificity, versatility and feasibility, DW MRI protocols are employed in assessing skull lesions. If a lesion such as ours is encountered in everyday practice, a differential diagnosis of a benign etiology should also be considered. 


\section{References}

[1] Patel SR, Benjamin RS. Chapter 98. Soft Tissue and Bone Sarcomas and Bone Metastases. In: Longo DL, Fauci AS, Kasper DL,Hauser SL, Jameson JL, Loscalzo J, eds. Harrison's Principles of Internal Medicine. 18th ed. New York: McGraw-Hill; 2012: 712-724.

[2] Edwards E, Desouza M. Diffusion weighted MRI and its application to cancer. Cancer Imaging. 2006; 6: 135-143. PMid:17015238 http://dx.doi.org/10.1102/1470-7330.2006.0021

[3] Win AZ, Aparici CM. Examining the value PSA=10ng/ml as a cutoff for predicting metastatic bone disease in NaF18 PET/CT bone scans, a pilot study. Journal of Biomedical Graphics and Computing. 2012; 2: 57-64. http://dx.doi.org/10.5430/jbgc.v2n1p57

[4] Nemeth AJ, Henson JW, Mullins ME, Gonzalez RG, Schaefer PW. Improved detection of skull metastasis with DiffusionWeighted MR Imaging. Am J Neuroradiol. 2007; 28: 1088-1092. PMid:17569964 http://dx.doi.org/10.3174/ajnr.A0501

[5] Thoeny HC, De Keyzer F, King AD. Diffusion-Weighted MR Imaging in the head and neck. Radiology. 2012; 263: 19-24. PMid:22438440 http://dx.doi.org/10.1148/radiol.11101821

[6] Ghanem N, Uhl M, Brink I, Schäfer O, Kelly T, Moser E, Langer M. Diagnostic value of MRI in comparison to scintigraphy, PET, MS-CT and PET/CT for the detection of metastases of bone. Eur J Radiol. 2005 Jul; 55(1): 41-55. PMid:15950100

[7] Mosavi F, Johansson S, Sandberg DT, Turesson I, Sörensen J, Ahlström H. Whole-body diffusion-weighted MRI compared with (18)F-NaF PET/CT for detection of bone metastases in patients with high-risk prostate carcinoma. AJR Am J Roentgenol. 2012 Nov; 199(5): 1114-20. PMid:23096187 http://dx.doi.org/10.2214/AJR.11.8351

[8] Cook GJ, Fogelman I. The role of nuclear medicine in monitoring treatment in skeletal malignancy. Semin Nucl Med. 2001 Jul; 31(3): 206-11. PMid:11430527 http://dx.doi.org/10.1053/snuc.2001.23527

[9] Kawaguchi M, Tateishi U, Shizukuishi K, Suzuki A, Inoue T. 18F-fluoride uptake in bone metastasis: morphologic and metabolic analysis on integrated PET/CT. Ann Nucl Med. 2010 May; 24(4): 241-7. PMid:20333485

http://dx.doi.org/10.1007/s12149-010-0363-0

[10] Laurent V, Trausch G, Bruot O, Olivier P. Comparative study of two whole-body imaging techniques in the case of melanoma metastases. European Journal of Radiology. 2010; 75: 376-383. PMid:19497694 http://dx.doi.org/10.1016/j.ejrad.2009.04.059

[11] Cai M, Ma L, Xu, G, Gruen P, Li J, Yang M, Pan L, et al. Clinical and radiological observation in a surgical series of 36 cases of fibrous dysplasia of the skull. Clinical Neurology and Neurosurgery. 2012; 114: 254-259. PMid:22088360 http://dx.doi.org/10.1016/j.clineuro.2011.10.026

[12] Lee YG, Cho CW. Benign osteoblastoma located in the parietal bone. J Korean Neurosurg Soc. 2010; 48: 170-172. PMid:20856669 http://dx.doi.org/10.3340/jkns.2010.48.2.170

[13] Scutellari PN, Giorgi A, De Sario V, Campanati P. Correlation of multimodality imaging in Paget's disease of bone. Radiol Med. 2005; 110: 603-615.

[14] Adams A, Offiah C. Central skull base osteomyelitis as a complication of necrotizing otitis externa: Imaging findings, complications, and challenges of diagnosis. Clin Radiol. 2012; 67: e7-e16. PMid:22483946 http://dx.doi.org/10.1016/j.crad.2012.02.004 